\title{
Extramedullary Plasmacytoma of the Nasopharynx Treated with Surgery and Adjuvant Radiation: Case Report and Review of the Literature
}

\author{
J Leibowitz, M Cohen, N Hashmi, N Mirza, S Abboud
}

\section{Citation}

J Leibowitz, M Cohen, N Hashmi, N Mirza, S Abboud. Extramedullary Plasmacytoma of the Nasopharynx Treated with Surgery and Adjuvant Radiation: Case Report and Review of the Literature. The Internet Journal of Otorhinolaryngology. 2009 Volume 12 Number 2.

DOI: $\underline{10.5580 / \mathrm{b} 95}$

\begin{abstract}
Purpose: Extramedullary plasmacytoma (EMP) is a rare neoplasm of plasma cell origin. Although the head and neck is the most common location of EMP, there are only a handful of cases reported in the nasopharynx. Design: Case report and review of the literature

Results: A 50 year-old healthy male presented with a year-long history of increasing bilateral nasal congestion unresponsive to medical therapy. Nasal endoscopy revealed a large exophytic mass filling both posterior nasal cavities. Imaging demonstrated a $3 X 3 \mathrm{~cm}$ mass arising from the nasopharynx and extending into the posterior nasal cavities bilaterally. A biopsy was performed, demonstrating extramedullary plasmacytoma. The patient underwent surgical debulking followed by radiation therapy to the primary site and bilateral necks. He has not recurred to date.

Conclusions: Although surgery is usually considered second line treatment for EMP of the head and neck, this case demonstrates the utility of surgical debulking in symptom palliation before definitive treatment with radiation.
\end{abstract}

\section{INTRODUCTION}

Extramedullary plasmacytoma (EMP) is a rare neoplasm of plasma cell origin, and is diagnosed when there is a focus of monoclonal plasma cells in the soft tissue in the absence of systemic disease. EMP is characterized as a plasma cell tumor, along with solitary plasmacytoma of bone and multiple myeloma, which is considered a more advanced stage of disease. EMP accounts for 3-4\% of all plasma cell tumors. ${ }^{1,2}$ The majority of cases occur between the $4{ }^{\text {th }}$ and 7 th decade of life ${ }^{2}$, with a 2.6-3:1 male dominance. ${ }^{1,2,3,15}$ The most common location is the head and neck, with estimates ranging up to $80-90 \% .^{2,3,4}$ The nasal cavity is the most common location, followed by the paranasal sinuses, nasopharynx, oropharynx and larynx. ${ }^{1,2}$ Symptoms are nonspecific, and are due to local tumor effects. They include nasal congestion, anosmia, hyposmia, epistaxis, rhinnorrhea, pain, and neck swelling. There are only a handful of reports specifically describing EMP arising in the nasopharynx. This report conveys our recent experience with EMP of the nasopharynx in the context of the limited literature on this topic, with an emphasis on multimodality treatment and symptom palliation with surgery.

\section{CASE REPORT}

A 50 year-old healthy, African-American male presented with a year-long history of progressive bilateral nasal congestion unresponsive to medical therapy (including nasal saline irrigations, topical steroid sprays, and nasal decongestants), in the absence of other nasal or systemic complaints. Physical exam was normal. Nasal endoscopy revealed a large exophytic mass arising from the nasopharynx, filling both choanae and extending into the nasal cavities bilaterally. CT scan revealed a $3 X 3 \mathrm{~cm}$, enhancing mass arising from the posterior nasopharynx and extending into both posterior nasal cavities. (Figure $1 \& 2$ ) 


\section{Figure 1}

FIGURE 1: CT scan with contrast of nasopharynx. Axial image demonstrating a contrast enhancing mass in the nasopharynx extending into the posterior nasal cavities bilaterally. Focal areas of hypodensity indicate necrosis.

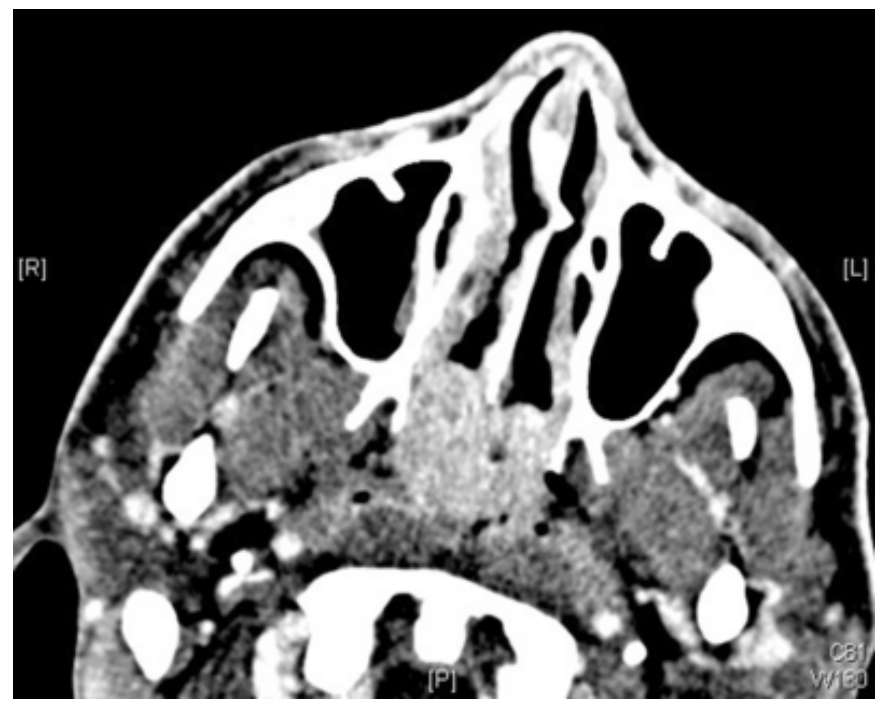

Figure 2

FIGURE 2: CT scan with contrast of nasopharynx. Coronal Image.

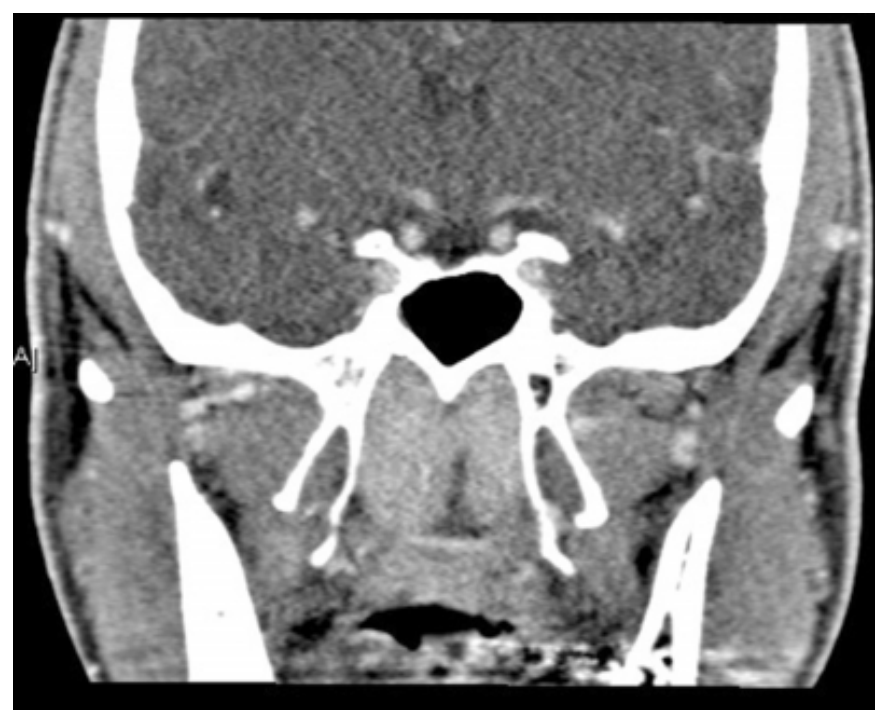

The patient subsequently underwent biopsy in the operating room to obtain tissue diagnosis and further characterize the lesion. Pathologic sections showed nasopharyngeal mucosa with squamous metaplasia and an underlying monotonous infiltrate of sheets of plasma cells with mild cytologic atypia and occasional binucleation. (Figure $3 \& 4$ )

\section{Figure 3}

FIGURE 3: The tumor cells are Lambda light chain restricted (upper) and positive for CD 138 (lower), 10X magnification.
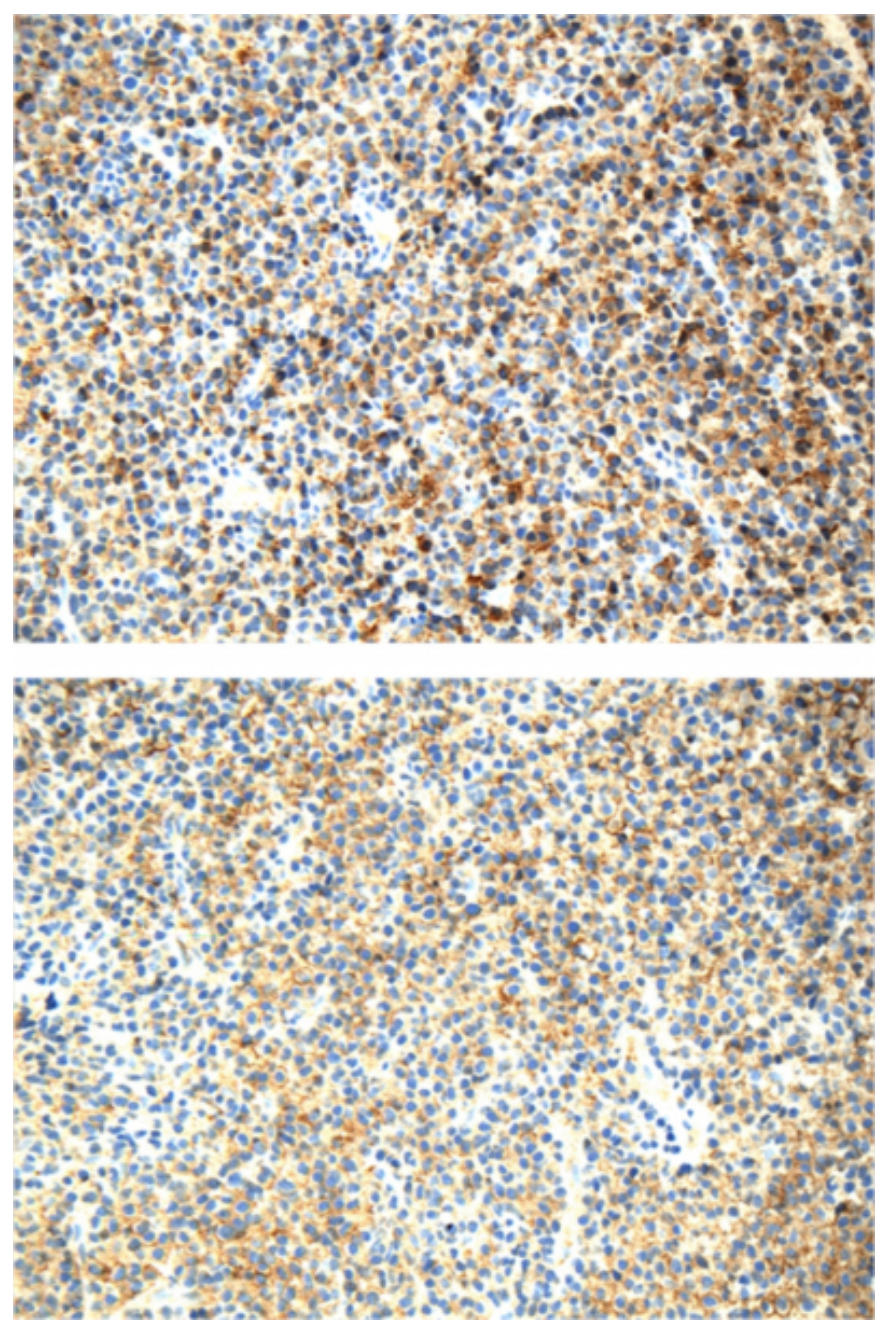


\section{Figure 4}

FIGURE 4: The tumor consists of sheets of plasma cells with atypia $10 \mathrm{X}$ magnification.

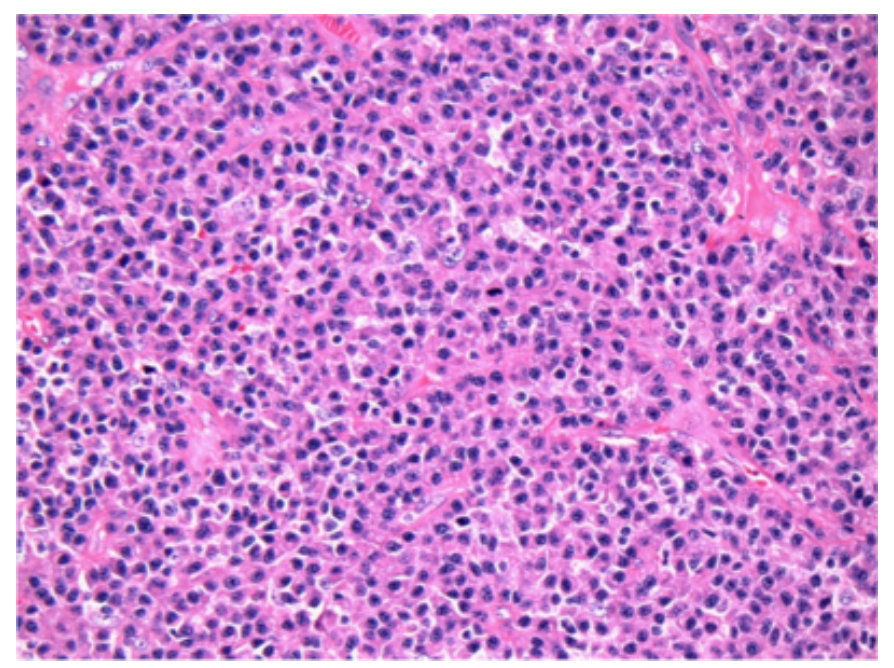

Immunostains showed an expansion of IgG lambda restricted CD138+ plasma cells. Flow cytometry performed on representative tissue demonstrated unremarkable T cells, NK cells and monocytes. Hematological work-up revealed no other lesions. Serum and urine protein electrophoresis and immunofixation failed to reveal evidence of a paraprotein. Levels of IgG and IgA were within normal limits. Bone marrow biopsy failed to demonstrate evidence of a clonal plasma cells. Skeletal survey was negative. These findings were consistent with a diagnosis of extramedullary plasmacytoma.

Given the size and location of the lesion, with its attendant morbidity from near total nasal obstruction, as well as the expected worsening of the patient's nasal obstruction due to edema from radiation, the patient underwent bulk endoscopic resection prior to definitive treatment with radiation. Prior to radiation (but after surgical debulking), a PET-CT was obtained, and was concerning for PET-avid bilateral level III lymph nodes. Radiation therapy consisted of external beam radiation given as 50 Gy over 5 weeks, with bilateral draining nodes included in the fields. The patient has been disease free for 19 months with no evidence of loco-regional recurrence or multiple myeloma.

\section{DISCUSSION}

The diagnosis of extramedullary plasmacytoma (EMP) is made when a localized collection of monoclonal plasma cells exists in the absence of plasma cell proliferation elsewhere in the body (i.e. bone marrow, blood). Diagnostic criteria, as recommended by the UK Myeloma Forum include: Single extramedullary mass of clonal plasma cells, histologically normal bone marrow aspirate, normal skeletal survey, no evidence of anemia, hypercalcemia, or renal impairment due to myeloma, and absent or low serum and urinary monoclonal immunoglobulin. ${ }^{3}$ In less than $25 \%$ of patients, a monoclonal paraprotein can be detected in the blood or urine. Systemic evaluation should include CBC, ESR, Serum and urine protein electrophoresis, immunoglobulin determination, bone marrow biopsy, and skeletal survey. ${ }^{1}$ Others recommend spinal MRI for staging to evaluate for bony lesions. ${ }^{5}$ The goal of testing is to exclude systemic involvement characteristic of multiple myeloma. Some authors also recommend pan-endoscopy for suspected cases of EMP, including nasal endoscopy, laryngoscopy, tracheo-bronchoscopy, and esophagoscopy/gastrocopy. ${ }^{2}$

CT or MRI is critical to delineate the extent of local disease $\mathrm{e}^{2,3}$; however, there are no characteristic imaging criteria. ${ }^{6}$ Due to the submucosal nature of the tumor, deep biopsies or excisional biopsies are sometimes required for diagnosis; fine needle aspiration may be inadequate., ${ }^{2,37,8}$ Histologically, EMP may mimic other conditions, such as lymphoma, undifferentiated carcinoma, reactive plasmacytoma, and plasma cell granuloma; these are best differentiated by phenotypic studies that show a CD 38+ clonal population as demonstrated by cytoplasmic light chain restriction. ${ }^{4,5}$

\section{EMP AND THE NASOPHARYNX (NP)}

Between 1905 and 1997, 131 cases of EMP involving the nasopharynx have been reported, accounting for approximately $18 \%$ of cases of the head and neck. ${ }^{2}$ More recently, Wein et al reviewed 21 cases of EMP of the NP, reporting a multiple myeloma progression rate of $9.5 \% .^{13}$ Unfortunately, these reports do not specify the exact treatment for the nasopharynx. There are also handful of reports detailing surgical excision of limited nasopharyngeal EMP followed by adjuvant radiation with good results. ${ }^{14}$

\section{RADIATION}

Given its radiosensitivity, radiation is the first line treatment for EMP, with local cure rates of $80-100 \%$ the norm.

However, given the retrospective nature of the literature, there is no evidence based dosing regimen, with most larger series reporting the use of $35-60 \mathrm{~Gy} .{ }^{3,4,8,9,10}$ The most recent recommendations of the UK Multiple Myeloma Forum range from 40-50 Gy depending on the size of the tumor. 
The role of chemotherapy is not clearly defined; currently its use is limited to select tumors $>5 \mathrm{~cm}$, high grade tumors, or residual/recurrent disease. ${ }^{3}$

\section{SURGERY}

Traditionally, surgery alone has had a limited role in the treatment of EMP, and its use remains controversial.

Although surgery can be curative, there is significant morbidity associated with adequate resection. Further, it is not always possible to obtain adequate margins in the head and neck. Given these limitations, as well as the responsiveness of EMP to radiation, surgery is currently a second line treatment. In a review of 714 cases of EMP of the head and neck, Alexoui et al. found that $44.3 \%$ of cases were treated with radiation alone, while $26.9 \%$ were treated with surgery \& radiation, and $21.9 \%$ with surgery alone. They found a statistically significant increase in overall or recurrence free survival in patients treated with surgery and radiation. ${ }^{2}$ When resectable, they recommend surgical resection; adjuvant radiation is utilized in cases of close margins or incomplete resection. ${ }^{2}$ This position is supported by other authors. ${ }^{1,8}$

In terms of the nasopharynx there are no reports directly addressing surgical resection, although some authors have utilized endoscopic or open methods to resect such lesions. ${ }^{11,12}$ Our case is unique in that surgery was used both for diagnosis and to debulk the lesion to improve quality of life. Upon endoscopic removal of the lesion, the patient had a significant and immediate improvement in his quality of life as a result of his immediate relief of complete nasal obstruction.

Another controversy in the literature is the need for prophylactic treatment of the draining lymph nodes. Rates of reported nodal spread vary in the literature, from as low as $5-10 \%$ to as high as $40 \%{ }^{1,2,3}$ Furthermore, different authors have achieved excellent results when clinically negative necks have either been included or excluded from the radiation fields. Currently, the UK myeloma forum recommends treating regional nodes if they are clinically involved or if the nodes are regarded as being at high risk (i.e. primary site in Waldeyers Ring). ${ }^{3}$ In our patient, the necks were included in the radiation fields due to concern for regional spread.

\section{PROGNOSIS}

Overall, EMP carries a favorable prognosis. Most authors report a local recurrence rate after treatment of less than
$20 \%$, with some reporting rates of $5-10 \% .^{1,2,4,5,16}$ Prognostic factors have not been clearly delineated, although tumor size $^{10,16}$ and older age ${ }^{15}$ have been reported as negative prognostic factors. Some authors have suggested improved local control with radiation (either with or without surgery). ${ }^{1}$ Reported 5-year survival rates for EMP range from $70-79 \%{ }^{15}$, while 10 -year survival rates are $72 \% .^{16}$

Conversion to multiple myeloma usually occurs approximately 2-3 years after diagnosis. ${ }^{3,15}$ Rates of conversion to $\mathrm{MM}$ in the literature are reported as less than $35-45 \%$, although some larger series have reported rates of approximately $15 \% .^{1,2,4,7,16}$ There is some evidence that rates of progression to MM are higher in patients treated with radiation $^{2}$, although others have not seen this relationship. ${ }^{1}$ In patients who develop MM, disease free and overall survival range from $50-80 \%$ at 10 years. ${ }^{5}$ Survival $>10$ years after diagnosis is common.

\section{CONCLUSIONS}

This report adds to the scant literature on EMP of the nasopharynx, which may mimic many benign nasal and sinus conditions, and must be included in the differential diagnosis of refractory nasal obstruction and congestion. Although surgery is usually considered second line treatment for EMP of the head and neck, this case demonstrates the utility of surgical debulking in symptom palliation before definitive treatment with radiation.

\section{References}

1. Bachar G, Goldstein D, Brown D, et al: Solitary extramedullary plasmacytoma of the head and neck - Longterm outcome analysis of 68 cases. Head \& Neck; 2008; 30:1012-1019

2. Alexiou C, Kau R, Dietzfelbinger H, et al: Extramedullary Plasmacytoma tumor occurrence and therapeutic concepts. Cancer; 1999;85:2305-2314

3. Soutar R, Lucraft H, Jackson G, et al: Guidelines on the diagnosis and management of solitary plasmacytoma of bone and solitary extramedullary plasmacytoma. British J of Haematology 2004; 124,717-726

4. Liebross RH, Cox J, Weber D, Delasalle K, Alexanian R: Clinical course of solitary extramedullary plasmacytoma.

Radiotherapy and Oncology; 1999; 52:245-249

5. Weber DM: Solitary Bone and extramedullary plasmacytoma. Hematology; 2005; 373-376

6. Ching ASC, Khoo JBK, Chong VFH: CT \& MR imaging of solitary extramedullary plasmacytoma of the nasal tract. Am J Neuroradiol; 2002; 23:1632-1636

7. Nofsinger YC, Mirza N, Rowan PT, Lanza D, Weinstein GS: Head \& neck manifestations of plasma cell neoplasms. Laryngoscope; 1997: 107:741-746

8. Miller FR, Lavertu P, Wanamaker JR, Bonafeded J, Wood BG: Plasmacytomas of the head and neck. Otolaryngology-

Head and Neck Surgery; 1998; 119:614-618

9. Susnerwala SS, Shanks JH, Banerjee SS, Scarfee JH,

Farrington WT, Slevin NJ: Extramedullary plasmacytoma of 
the head and neck region: clinicopathologic correlation in 25 cases. Br J of Cancer; 1997; 75:921-927

10. Tsang RW, Gospodarowicz MK, Pintilie M, et al: Solitary plasmacytoma treated with radiotherapy: Impact of tumor size on outcome. Int J Radiation Oncology Biol. Phys; 2001; 50:113-120

11. Baek BJ, Kim SW, Park H, Park JK, Han KY, Oh CH:

Extramedullary plasmacytoma arising from the nasal septum. ENT J; 2005; 84:720-722

12. Sadek SAA, Dogra TS, Khan MK, Baraka ME, Sidhom NN: Plasmacytoma of the nasopharynx. The Journal of Laryngology \& Otology; 1985;99:1289-1292

13. Wein RO, Popat SR, Doerr TD, Dutcher PO: Plasma cell tumors of the skull base: four cases reports and literature review. Skull Base; 2002; 12:77-86

14. J. Clinical Oncology; 2008; 26: 5817-5819

15. Dores GM, Landgren O, McGkynn KA, Curtis RE, Linet MS, Devesa SS: Plasmacytoma of bone, extramedullary plasmacytoma and multiple myeloma: incidence and survival in the United States 1992-2004. British J Haematology; 2008; 144:86-94.

16. Ozsahin M, Tsang RW, Poortmans P, et al: Outcomes and patterns of failure in solitary plasmacytoma: a multicenter rare cancer network study of 258 patients. Int $\mathbf{J}$ Radiation Oncology Biol. Phys; 2006; 64: 210-217 


\section{Author Information}

Jason M. Leibowitz, MD

Department of Otorhinolaryngology/Head and Neck Surgery, University of Pennsylvania Health System

\section{Marc A. Cohen, MD}

Department of Otorhinolaryngology/Head and Neck Surgery, University of Pennsylvania Health System

\section{Nazish Hashmi, MD}

Department of Otorhinolaryngology/Head and Neck Surgery, University of Pennsylvania Health System

\section{Natasha Mirza, MD}

Department of Otorhinolaryngology/Head and Neck Surgery, University of Pennsylvania Health System

\section{Soo K. Abboud, MD}

Department of Otorhinolaryngology/Head and Neck Surgery, University of Pennsylvania Health System 